\title{
In vitro Infectivity of Strains Isolated From Dogs Naturally Infected With Leishmania infantum Present a Distinct Pathogenic Profile in Hamsters
}

Lucilene Aparecida Resende ${ }^{1,2}$, Rodrigo Dian de Oliveira Aguiar-Soares ${ }^{2}$, Nádia das Dores Moreira ${ }^{2,3,4}$, Sidney de Almeida Ferreira ${ }^{2,5}$, Mariana Ferreira Lanna ${ }^{1,2}$, Jamille Mirelle de Oliveira Cardoso ${ }^{2}$, Fernando Augusto Siqueira Mathias ${ }^{2}$,

OPEN ACCESS

Edited by:

Richard V. Goering,

Creighton University, United States

Reviewed by:

Thiago DeSouza-Vieira

National Institutes of Health (NIH),

United States

Valter Viana Andrade-Neto,

Oswaldo Cruz Foundation

(Fiocruz), Brazil

*Correspondence:

Rodolfo Cordeiro Giunchetti giunchetti@icb.ufmg.br; giunchetti@gmail.com

Specialty section:

This article was submitted to Infectious Diseases Surveillance,

Prevention and Treatment a section of the journal

Frontiers in Medicine

Received: 28 February 2020

Accepted: 20 July 2020

Published: 28 August 2020

Citation

Resende LA, Aguiar-Soares RDdO, Moreira NdD, Ferreira SdA, Lanna MF,

Cardoso JMdO, Mathias FAS,

Coura-Vital W, Mariano RMdS, Leite JC, Silveira P, de Carvalho TF

Santos RL, Silveira-Lemos Dd,

Martins-Filho OA, Dutra WO, Reis AB and Giunchetti RC (2020) In vitro

Infectivity of Strains Isolated From

Dogs Naturally Infected With

Leishmania infantum Present a

Distinct Pathogenic Profile in

Hamsters. Front. Med. 7:496.

doi: 10.3389/fmed.2020.00496
Wendel Coura-Vital 2,3,4,6, Reysla Maria da Silveira Mariano ${ }^{1}$, Jaqueline Costa Leite ${ }^{1}$, Patricia Silveira ${ }^{1}$, Tatiane Furtado de Carvalho ${ }^{7}$, Renato Lima Santos ${ }^{7}$, Denise da Silveira-Lemos ${ }^{1,8,9}$, Olindo Assis Martins-Filho ${ }^{8}$, Walderez Ornelas Dutra ${ }^{1}$, Alexandre Barbosa Reis ${ }^{2,3,4}$ and Rodolfo Cordeiro Giunchetti ${ }^{\text {* }}$

${ }^{1}$ Laboratório de Biologia das Interações Celulares, Departamento de Morfologia, Instituto de Ciências Biológicas, Universidade Federal de Minas Gerais, Belo Horizonte, Brazil, ${ }^{2}$ Laboratório de Imunopatologia, Núcleo de Pesquisas em Ciências Biológicas, Universidade Federal de Ouro Preto, Ouro Preto, Brazil, ${ }^{3}$ Departamento de Análises Clínicas, Escola de Farmácia, Universidade Federal de Ouro Preto, Ouro Preto, Brazil, ${ }^{4}$ Programa de Pós Graduação em Ciências Farmacêuticas (Cipharma), Escola de Farmácia, Universidade Federal de Ouro Preto, Ouro Preto, Brazil, ${ }^{5}$ Laboratório de Biologia Parasitária, Faculdade de Ciências da Saúde, Universidade Federal de Lavras, Lavras, Brazil, ${ }^{6}$ Laboratório de Pesquisa em Epidemiologia e Citologia, Departamento de Análises Clínicas, Escola de Farmácia, Universidade Federal de Ouro Preto, Ouro Preto, Brazil, ' Departamento de Clínica e Cirurgia Veterinárias, Escola de Veterinária, Universidade Federal de Minas Gerais, Belo Horizonte, Brazil, ${ }^{8}$ Grupo Integrado de Pesquisas em Biomarcadores, Instituto René Rachou, FIOCRUZ, Belo Horizonte, Brazil, ${ }^{9}$ Departamento de Medicina, Universidade José Do Rosário Vellano, UNIFENAS, Belo Horizonte, Brazil

Visceral leishmaniasis $(\mathrm{VL})$ is a severe disease caused by Leishmania infantum. Dogs are the parasite's main reservoir, favoring its transmission in the urban environment. The analysis of $L$. infantum from infected dogs contributes to the identification of more virulent parasites, thereby supporting basic and applied studies such as vaccinal and therapeutic strategies. We proposed the in vitro and in vivo characterization of $L$. infantum strains from naturally infected dogs from a VL endemic area based on an infectivity and pathogenicity analysis. DH82 canine macrophages were infected in vitro with different strains for infectivity analysis, showing distinct infectivity profiles. The strains that showed greater and lesser infectivity using in vitro analyses (616 and 614, respectively) were used to infect hamsters for pathogenicity analysis. The group infected with strain 616 showed $100 \%$ survival while the group infected with strain 614 showed $50 \%$ after seven months of follow up. Furthermore, the 614 strain induced more noticeable clinicopathological changes and biochemical abnormalities in liver function, along with high inflammation and parasite load in the liver and spleen. We confirmed high variability of infectivity and pathogenicity in $L$. infantum strains from infected dogs. The results support the belief that screening for $L$. infantum infectivity using in vitro experiments is inadequate when it comes to selecting the most pathogenic strain.

Keywords: Leishmania infantum, visceral leishmaniasis, experimental infection, pathogenicity, infectivity 


\section{INTRODUCTION}

Leishmania infantum (syn. Leishmania chagasi) is the etiological agent of visceral leishmaniasis (VL) in Latin America, a severe chronic systemic disease (1-3). More than $90 \%$ of VL cases reported in the New World occur in Brazil. Initially, VL presented a rural epidemiological profile, but it has expanded into urban areas in the past few decades $(4,5)$.

Dogs are the main domestic reservoir for the parasite in various geographical locations, playing an important role in its transmission to humans $(6,7)$. Moreover, euthanasia has been shown to have limited effects in controlling visceral leishmaniasis in Brazil (8).

A major problem in controlling the transmission of visceral leishmaniasis in endemic areas is that many dogs are asymptomatic, even when presenting high levels of skin parasitism (9, 10). Thus, asymptomatic dogs are highly competent transmitters of $L$. infantum, making canine visceral leishmaniasis (CVL) more important epidemiologically than human VL $(9,11,12)$.

Manifestation of the disease depends on the virulence of the Leishmania strain, as well on the genetic background and immune status of the host (13-16). A study conducted by Cunha et al. (17) characterized the biology and infectivity of viscerotropic and dermatotropic strains of $L$. infantum isolated from $\mathrm{HIV}+$ and HIV- patients in the VL murine model, and demonstrated that in vivo and in vitro virulence are intrinsic characteristics of each strain. Another study that evaluated the ex vivo virulence of the L. infantum isolated from Phlebotomus perniciosus captured in an endemic area of Madrid, Spain, demonstrated that L. infantum strains exhibited high virulence, and were associated with cytokine production and enzymatic activities involved in the VL pathogenesis (18). Moreover, it has been shown that in vitro infection of human monocytes by Leishmania strains induced distinct immunological characteristics and the host cell's ability to control Leishmania (19). Based on these data, it is reasonable to hypothesize that parasite transmission in endemic areas could be related to distinct infectivity and virulence profiles.

In this study, we examined eight different $L$. infantum strains from dogs with CVL, characterizing them according to in vitro (L. infantum infectivity in DH82 cell linage) and in vivo (pathogenicity evaluation in Mesocrisetus auratus - hamster model) approaches. Our results clearly show that strains isolated from naturally infected dogs display distinct in vitro infectivity as well as pathogenic profiles in hamsters.

\section{MATERIALS AND METHODS}

\section{Ethical Approval}

The protocols for experimental L. infantum-infection in hamsters were approved by the Ethical Committee on Animal Research of the Universidade Federal de Ouro Preto, State of Minas Gerais, Brazil (approval ID number 2012/37).

\section{L. infantum Parasites Used for in vitro and in vivo Analysis}

Eight $L$. infantum strains were used in in vitro evaluations for this study: PP75 (WHO/MHOM/BR/74/PP75) and seven wild-type strains isolated from the bone marrow of naturally L. infantuminfected dogs from Belo Horizonte, Minas Gerais, as described in Table 1. All parasites used in this study were confirmed to be $L$. infantum by PCR-RFLP according to Coura-Vital et al. (20).

Parasites were grown in LIT and NNN (McNeal, Novy \& Nicolle) medium up to eight in vitro passages to preserve the most important parasite characteristics regarding infectivity and pathogenicity profiles, in addition to providing appropriate adaptation in the media culture. Stationary growth phase promastigotes, comprising predominantly metacyclic promastigotes (21-23), were obtained from an initial inoculum of $10 \mathrm{~mL}$ of LIT medium containing between $10^{7}$ and $10^{8}$ promastigotes $/ \mathrm{mL}$ in logarithmic growth. The promastigotes were added to $40 \mathrm{~mL}$ of NNN/LIT culture medium and stored at $23 \pm 1{ }^{\circ} \mathrm{C}$.

\section{In vitro Experimental Infection of Canine Macrophages}

The DF82 canine macrophage cell line (DS Pharma Biomedical, Osaka, Japan) was placed over circular coverslips $(15 \mathrm{~mm}$; Glasscyto, Brazil) on 24-well plates for $3 \mathrm{~h}$. Promastigotes, in stationary phase, were added at $4: 1$ and 10:1 parasite:host cell ratios. The cultures were incubated for 3,24 , and $48 \mathrm{~h}$ at $37^{\circ} \mathrm{C}$ in $5 \% \mathrm{CO}_{2}$. The amastigotes were counted in 300 macrophages using optical microscopy at $1,000 \mathrm{X}$ magnification. CFSE (Carboxyfluorescein succinimidyl diacetate ester; Vybrant ${ }^{\circledR}$ CFDA-SE Cell tracer kit, Invitrogen) was used to label parasites at a concentration of $2.8 \mathrm{~g} / \mathrm{mL}$ (24). After washing twice with

TABLE 1 | Clinical features of canine visceral leishmaniasis after isolation of $L$. infantum strains from naturally $L$. infantum-infected dogs.

\begin{tabular}{ll}
\hline Wild strains & $\begin{array}{l}\text { Clinical signs observed in dogs during L. infantum } \\
\text { isolation procedures }\end{array}$ \\
\hline 571 & Moderate weight loss \\
591 & Enlargement of popliteal lymph nodes, seborrheic \\
& dermatitis, ulceration in the left paw, jaundice, \\
& splenomegaly \\
& Intense weight loss, seborrheic dermatitis, periocular \\
& dermatitis, onychogryphosis, enlargement of \\
& pre-scapular lymph nodes \\
& Intense weight loss, seborrheic dermatitis, dermatitis in \\
& the periocular, ears, ribs and tail regions, \\
& onychogryphosis, enlargement of pre-scapular lymph \\
& nodes \\
& Moderate weight loss, seborrheic dermatitis, dermatitis \\
& in the periocular, ears, ribs and tail regions, \\
& onychogryphosis, enlargement of all lymph nodes \\
& Seborrheic dermatitis, dermatitis in the periocular, ears \\
& (with bleeding at the tip) and tail regions, localized \\
& ulceration, enlargement of all lymph nodes \\
& Moderate weight loss, dermatitis in the ears, \\
& onychogryphosis, enlargement of all lymph nodes \\
614 &
\end{tabular}


TABLE 2 | Hematological profile from non-infected animals (C; control group) and experimentally L. infantum-infected groups (reference strain: PP75; and wild strains: 614 and 616).

\begin{tabular}{|c|c|c|c|c|}
\hline \multirow{2}{*}{$\begin{array}{l}\text { Hematological } \\
\text { Parameters }\end{array}$} & \multicolumn{4}{|c|}{ Groups } \\
\hline & C & PP75 & 614 & 616 \\
\hline $\begin{array}{l}\text { Neutrophils } \\
\text { (cells/ } \mu \mathrm{L} \text { ) }\end{array}$ & $680.3 \pm 397.1$ & $951.6 \pm 582.3$ & $256.5 \pm 144.4$ & $812.2 \pm 585.5$ \\
\hline $\begin{array}{l}\text { Eosinophils } \\
\text { (cells/ } \mu \mathrm{L} \text { ) }\end{array}$ & $39.7 \pm 25.4$ & $67.0 \pm 46.2$ & $31.2 \pm 29.0$ & $50.4 \pm 38.1$ \\
\hline $\begin{array}{l}\text { Lymphocytes } \\
\text { (cells/ } \mu \mathrm{L})\end{array}$ & $2,012 \pm 659.4$ & $2,426 \pm 540.4$ & $1,568 \pm 1,011^{b}$ & $2,142 \pm 796.3$ \\
\hline $\begin{array}{l}\text { Monocytes } \\
\text { (cells/ } \mu \mathrm{L} \text { ) }\end{array}$ & $61.7 \pm 43.6$ & $65.4 \pm 35.6$ & $32.0 \pm 29.3$ & $65.6 \pm 76.6$ \\
\hline $\begin{array}{l}\text { Erythrocytes } \\
\left(10^{6} / \mu L\right)\end{array}$ & $6.4 \pm 1.1$ & $7.2 \pm 0.1$ & $3.9 \pm 2.1^{a, b, d}$ & $6.6 \pm 0.8$ \\
\hline $\begin{array}{l}\text { Hemoglobin } \\
\text { (g/dL) }\end{array}$ & $13.1 \pm 1.4$ & $14.0 \pm 0.5$ & $7.9 \pm 4.3^{a, b, d}$ & $13.1 \pm 1.7$ \\
\hline $\begin{array}{l}\text { Hematocrit } \\
\left(10^{6} / \mu \mathrm{L}\right)\end{array}$ & $27.7 \pm 2.9$ & $29.4 \pm 1.5$ & $17.1 \pm 8.6^{a, b, d}$ & $27.4 \pm 3.6$ \\
\hline VCM (fL) & $40.9 \pm 1.1$ & $40.8 \pm 1.0$ & $35.1 \pm 14.7^{a, b}$ & $41.3 \pm 1.0$ \\
\hline HCM (pg) & $19.4 \pm 0.6$ & $19.5 \pm 0.5$ & $16.1 \pm 6.7$ & $19.8 \pm 0.4$ \\
\hline
\end{tabular}

The measurements exhibited in the control group were used as reference values. Statistical differences were displayed as "a," "b," and "d" for the "C," "PP75," "616" groups, respectively. All data are shown as mean \pm standard deviation.

$15 \mathrm{~mL}$ RPMI, the pellet was suspended in $1 \mathrm{~mL}$ of RPMI 1640 and added in 4:1 and 10:1 parasite:host cell ratios. Labeled Leishmania were incubated together with macrophages in $\mathrm{CO}_{2}$ at $37^{\circ} \mathrm{C}$ for 3 and $24 \mathrm{~h}$ in polystyrene tubes (Becton Dickinson, Franklin Lakes, USA) to grow as non-adherent cells. Samples were centrifuged and ressuspended in $200 \mu \mathrm{L}$ of fix solution, MaxFacsFix (10.0 $\mathrm{g} / \mathrm{L}$ paraformaldehyde, $10.2 \mathrm{~g} / \mathrm{L}$ sodium cacodylate, and $6.65 \mathrm{~g} / \mathrm{L}$ sodium chloride, $\mathrm{pH}$ 7.2). A FACScan flow cytometer (Becton Dickinson, San Diego, USA) was used for acquisition, and data were analyzed using FlowJo ${ }^{\circledR}$ (FlowJo, LLC, Oregon, USA).

The in vitro experiments were used to select the most and least infective strains for use in a pathogenicity analysis in hamsters. The criteria used to select the parasites were based on: (i) the percentage of infection (low or high) consistently observed in optical microscopy and flow cytometry method, regardless of the difference in the intrinsic magnitude of data generated by microscopy and flow cytometry and (ii) isolates with intermediate infectivity patterns that were considered difficult to be categorized as most or least infective and, thus, not segregated into these categories.

\section{In vivo Experimental Infection in Hamsters}

Hamsters were experimentally infected with the wild strains chosen for presenting the lowest and highest infectivity profile after in vitro analysis, plus a reference strain (PP75). One-month-old male Syrian golden hamsters (Mesocricetus auratus), weighing $\sim 50-80 \mathrm{~g}$, were obtained from the Animal Science Center at the Universidade Federal de Ouro Preto (UFOP). Experimental infection was done at the end of seven days of promastigote cultivation [stationary phase presenting predominantly metacyclic forms as described by (23)] that were transferred to sterile polypropylene tubes (FalconH, Becton Dickinson, USA) and centrifuged at $900 \mathrm{xg}$ for $15 \mathrm{~min}$. The parasites were adjusted to $10^{7}$ promastigotes per inoculum. The experimental infection was made by intracardiac (i.c.) route, with the following strains ( $n=10$ /group): PP75 (WHO/MHOM/BR/74/PP75), 614, and 616 wild-type strains. Uninfected animals were used as a control group (C). Euthanasia was carried out 210 days after infection by injection, following anesthesia with intraperitoneal barbiturate (Thiopental ${ }^{\circledR}$ at 90 $\mathrm{mg} / \mathrm{kg}$ of body weight). Hamsters were clinically monitored throughout the experiment for signs of suffering due to Leishmania infection.

\section{Hematological and Biochemical Parameters in Hamster Model}

The hematological and biochemical parameters were analyzed as previously described (25). The samples were analyzed using the Auto Hematology Analyzer apparatus (Mindray BC-2800, Hamburg, Germany) for an overall analysis of leukocytes, erythrocytes, hematocrit, and hemoglobin. A differential cell count was performed on blood smears stained with Panotic Quick InstantProv $\left(\mathrm{Newprov}^{\circledR}\right)$ and evaluated by optical microscopy at 1,000X magnification. Biochemical analysis was based on evaluation of urea and creatinine levels for kidney function. Moreover, measures of the enzymes AST (aspartate aminotransferase) and ALT (alanine aminotransferase) were obtained to assess hepatic function. Biochemical analyses were performed using the Biochemical System Auto (CELM SBA200, Barueri, SP, Brazil) and commercials Labtest Kits (Labtest Diagnostica SA, Lagoa Santa, MG, Brazil), following the manufacturer's instructions.

\section{Histological Evaluation}

The size and appearance of the spleen and liver were macroscopically recorded during necropsy to check rounded edge and/or congestion resulting from organ enlargement and described as splenomegaly and hepatomegaly, respectively. Liver and spleen samples were fixed in $10 \%$ buffered formalin for $24 \mathrm{~h}$, paraffin embedded, and $4 \mu \mathrm{m}$ thick sections stained with hematoxylin and eosin (HE). Inflammatory, degenerative, and hyperplastic lesions were classified according to the following scores: 0 (absent), 1 (mild), 2 (moderate), and 3 (severe).

\section{Immunohistochemistry}

Immunohistochemistry was performed as previously described to evaluate parasite load (23) in sections of the spleen and liver (26). The numbers of immunolabeled Leishmania amastigotes in the spleen and liver sections were classified according to the following scores: 0 (absent), 1 (mild, 1-50 amastigotes per microscopic field under 400x magnification), 2 (moderate, 51100 per microscopic field under 400x magnification), and 3 (severe, more than 101 amastigotes per microscopic field under 400x magnification). 


\section{DNA Extraction and Quantitative Real-Time PCR}

DNA extraction and quantitative real time PCR were performed as previously described (21), using $20 \mathrm{mg}$ of tissue (spleen or liver). The concentration and purity of DNA was determined using spectrophotometer (NanoVue Plus, GE Healthcare Products, Piscataway, NJ, USA) based on $\mathrm{A}_{260} / \mathrm{A}_{280}$ and $\mathrm{A}_{260} / \mathrm{A}_{230}$ measures. To quantify parasite burdens, primers were used as described by Bretagne et al. (27), which amplified a 90-bp fragment of a single-copy of DNA polymerase gene of L. infantum (GenBank accession number AF009147). Standard curves were prepared for each run using known quantities of promastigotes (PP75 strain) seeded in cultures. Purified DNA was diluted from $10^{6}$ until 1.0 parasite/ $\mu \mathrm{L}$ following 1:10 ratio. Each curve point was performed in triplicate. The quantification cycles $(\mathrm{Cq})$ indicated the quantities of parasites per $20 \mathrm{ng}$ of total extracted DNA by interpolation of the standard curve. Afterwards, the total number of parasites was calculated for the total quantity of extracted DNA and divided by $20 \mathrm{mg}$ of tissue used in DNA purification. The final result was estimated as the number of amastigotes/mg of tissue. Reactions were processed and analyzed in an ABI Prism 7500-Sequence Detection System (Applied Biosystems, USA).

\section{Statistical Analysis}

Statistical analyses were conducted using Prism 5.0 software package (Prism Software, Irvine, CA, USA). Data normality was assessed using the Kolmogorov-Smirnoff test. One-way analysis of variance (ANOVA) and Tukey post-test were used to investigate differences between groups. Considering the nonparametric nature of real time PCR data, the Kruskal-Wallis test was used, followed by Dunn's test. Comparisons that returned a $P$-value $<0.05$ were considered statistically significant.

\section{RESULTS}

\section{The 616 Strain Presented a Highlighted Pattern of in vitro Infectivity}

It was observed that the 616 strain showed the highest percentage of infection and increased parasite load (Figures 1A-C). The analysis employing parasites labeled with CFSE was performed 3 and 24 hpi using the 1:4-1:10 parasite:macrophage ratios. The results confirmed that the 616 strain was the most infective, while the 614 strain was the least infective (Figures 1A-C). Figure 1D illustrates the interaction of parasites with macrophages by optical microscopy, showing the polarized profile as high and low infectivity in the 616 and 614 strains, respectively.

\section{Unlike the in vitro Experiments, the 614 Strain Displayed High Pathogenicity in Infected Hamsters Inducing $50 \%$ of the Deaths After 7 Months of Infection}

Seven months after experimental infection, the uninfected animals (C) and animals infected with the 616 strain showed

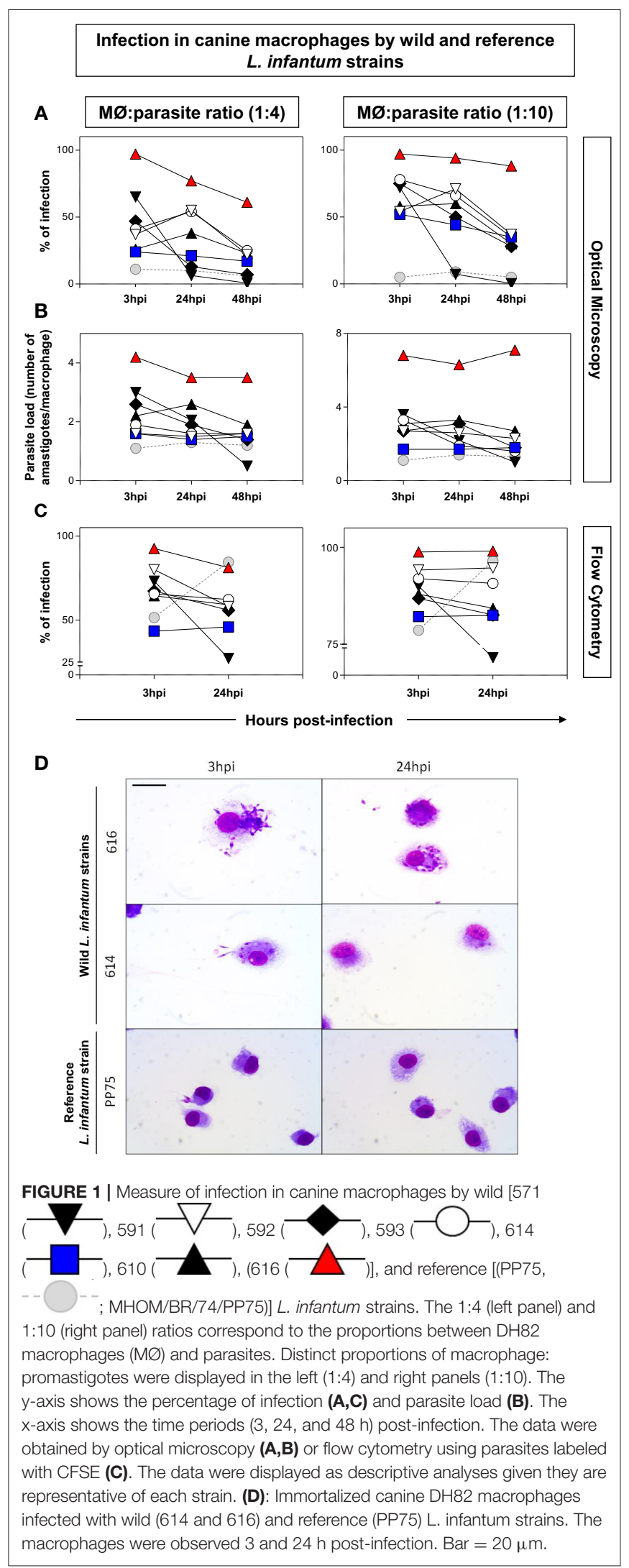


$100 \%$ survival rate. The animals infected with 614 strains showed a $50 \%$ survival rate at seven months postinfection (Figure 2), and exhibited more severe clinical signs (Figure 3). Moreover,

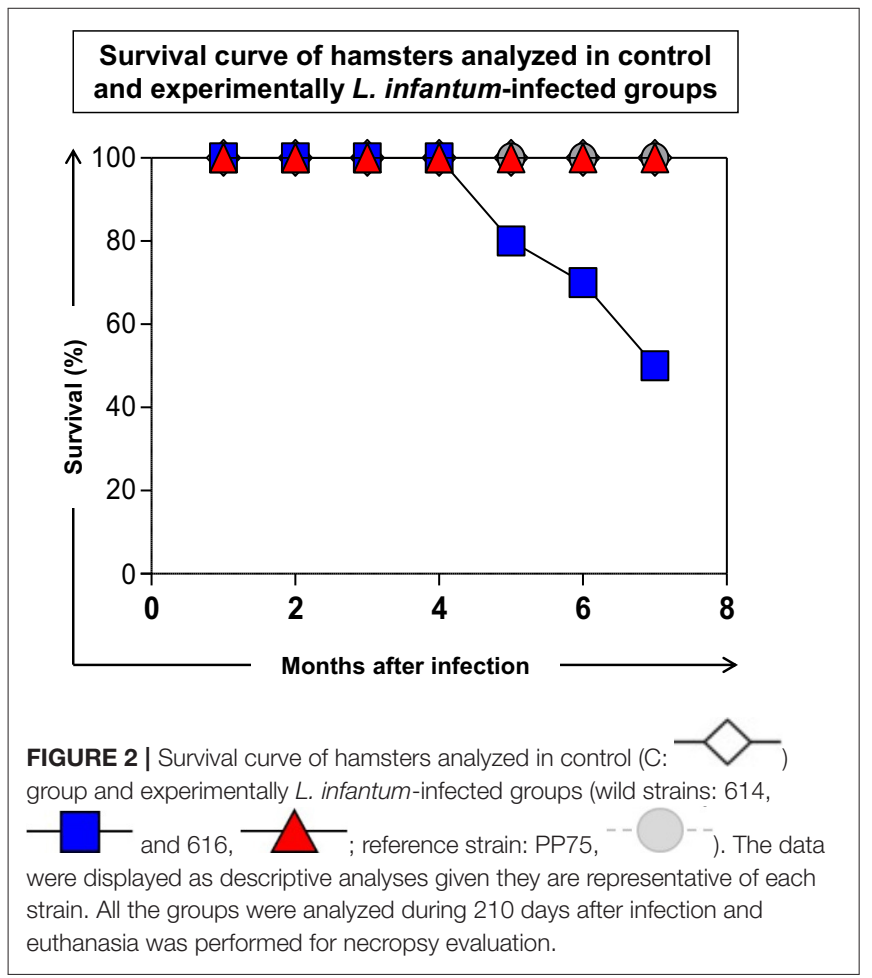

$60 \%$ of the animals infected with 614 strain had low weight and cachexia at the time of necropsy. In the group infected with 616 strain, 11 showed these signs (Figure 3A). The cachectic appearance in which these animals were found is illustrated in Figures 3B,C. All hamsters infected with 614 strain and $22 \%$ of 616 strain showed splenomegaly at the time of necropsy (Figure 3A).

\section{Hamsters Infected With the 614 Strain Exhibited Severe Anemia and Lymphopenia} Infection with the 614 strain induced severe anemia as observed by reduced levels $(P<0.05)$ of erythrocytes,

TABLE 3 | Biochemical profile from non-infected animals (C; control group) and experimentally L. infantum-infected groups (reference strain: PP75; and wild strains: 614 and 616)

\begin{tabular}{lcccc}
\hline \multirow{2}{*}{$\begin{array}{l}\text { Biochemical } \\
\text { Parameters }\end{array}$} & \multicolumn{4}{c}{ Groups } \\
\cline { 2 - 5 } & C & PP75 & $\mathbf{6 1 4}$ & $\mathbf{6 1 6}$ \\
\hline AST & $87.3 \pm 73.0$ & $83.8 \pm 30.1$ & $245.6 \pm 112.1^{\mathrm{a}, \mathrm{b}}$ & $163.6 \pm 117.6$ \\
ALT & $97.1 \pm 39.1$ & $78.4 \pm 19.2$ & $170.8 \pm 125.9$ & $134.1 \pm 50.1$ \\
Ureia & $47.6 \pm 3.8$ & $39.2 \pm 2.5$ & $94.3 \pm 96.8$ & $50.8 \pm 10.7$ \\
Creatinine & $0.4 \pm 0.0$ & $0.3 \pm 0.05$ & $0.3 \pm 0.1$ & $0.3 \pm 0.05$
\end{tabular}

The measurements exhibited in the control group were used as reference values. Statistical differences were displayed as "a" and "b," for the " $C$ " and "PP75" groups, respectively. All data are shown as mean \pm standard deviation.

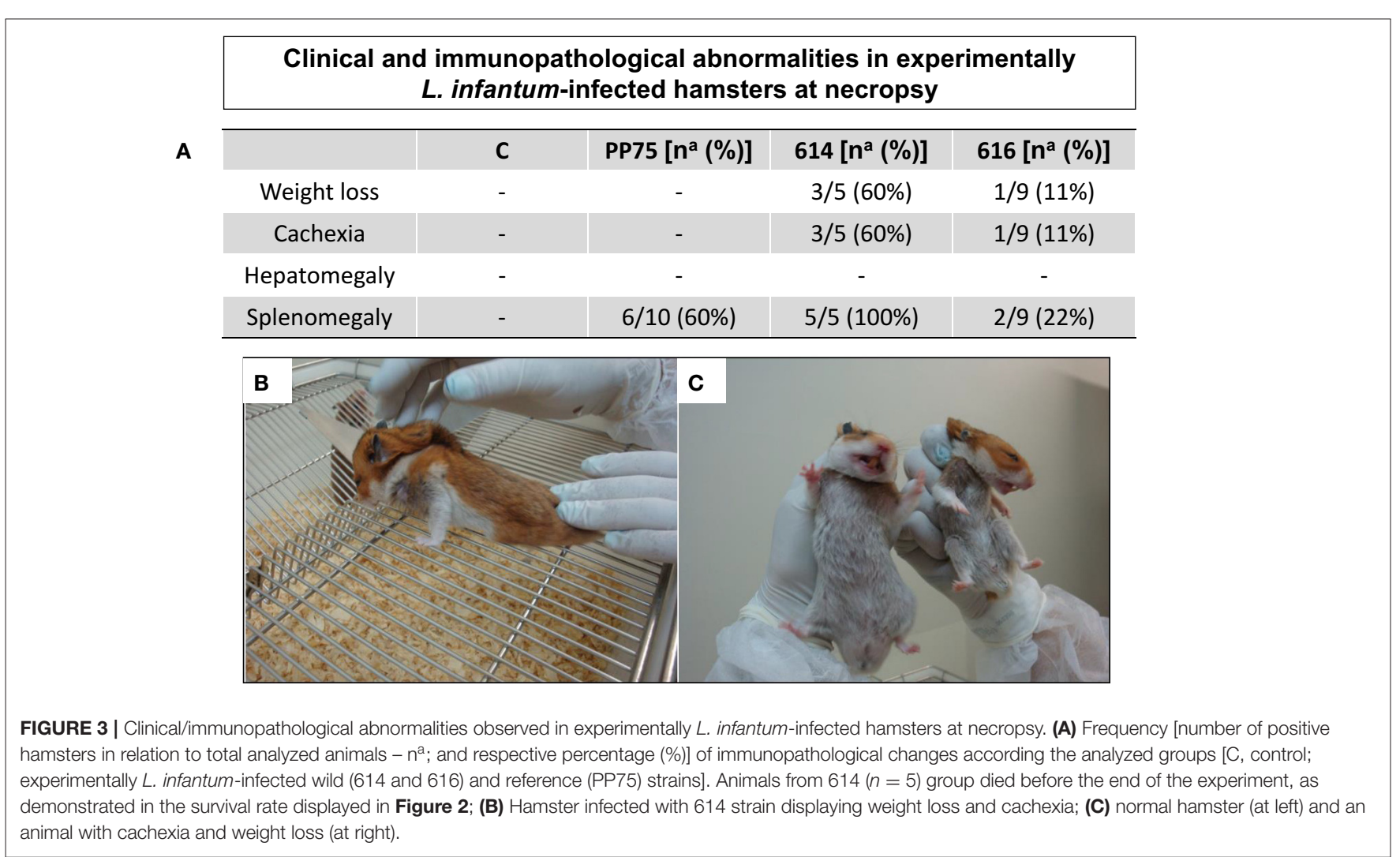




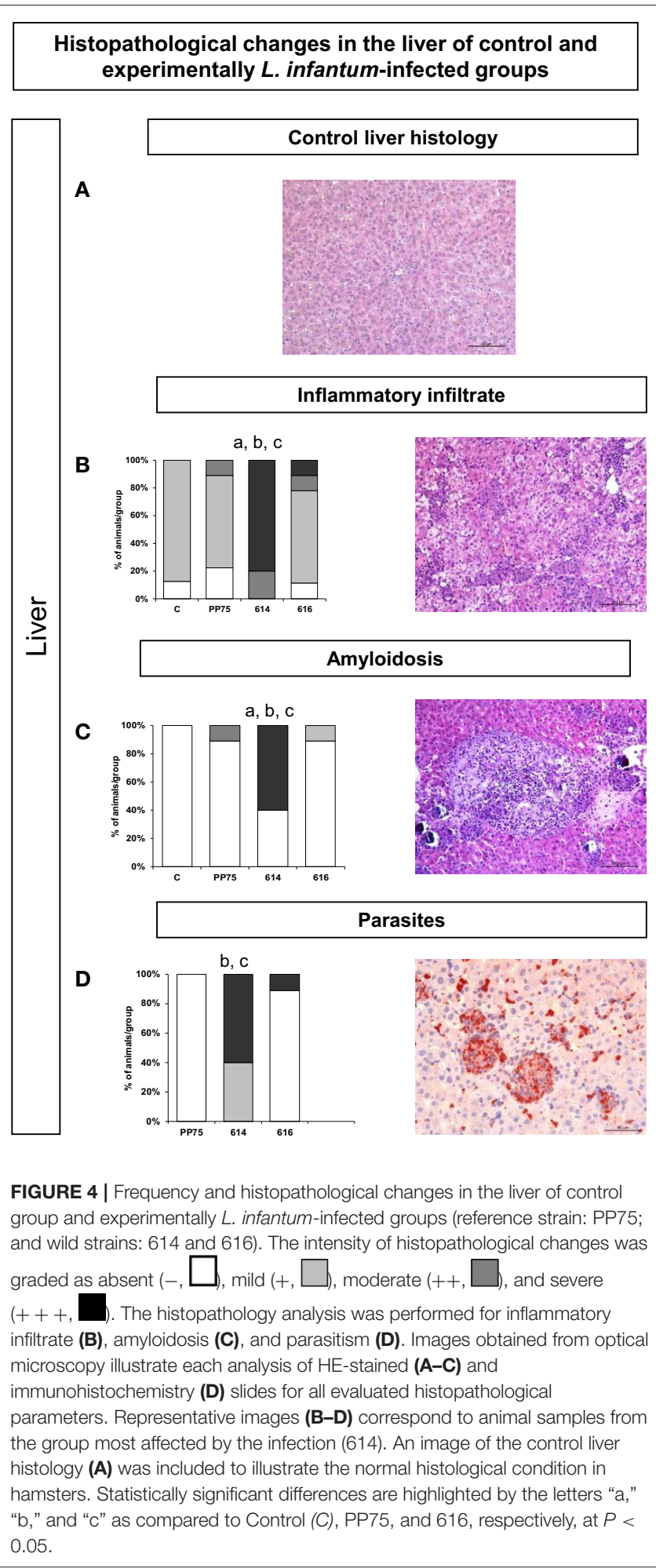

hemoglobin, and hematocrit, as compared to the other experimental groups (C, PP75, and 616) (Table 2). Moreover, infection with the 614 strain produced lower $(P<0.05)$ Histopathological changes in the spleen of control and
experimentally $L$. infantum-infected groups

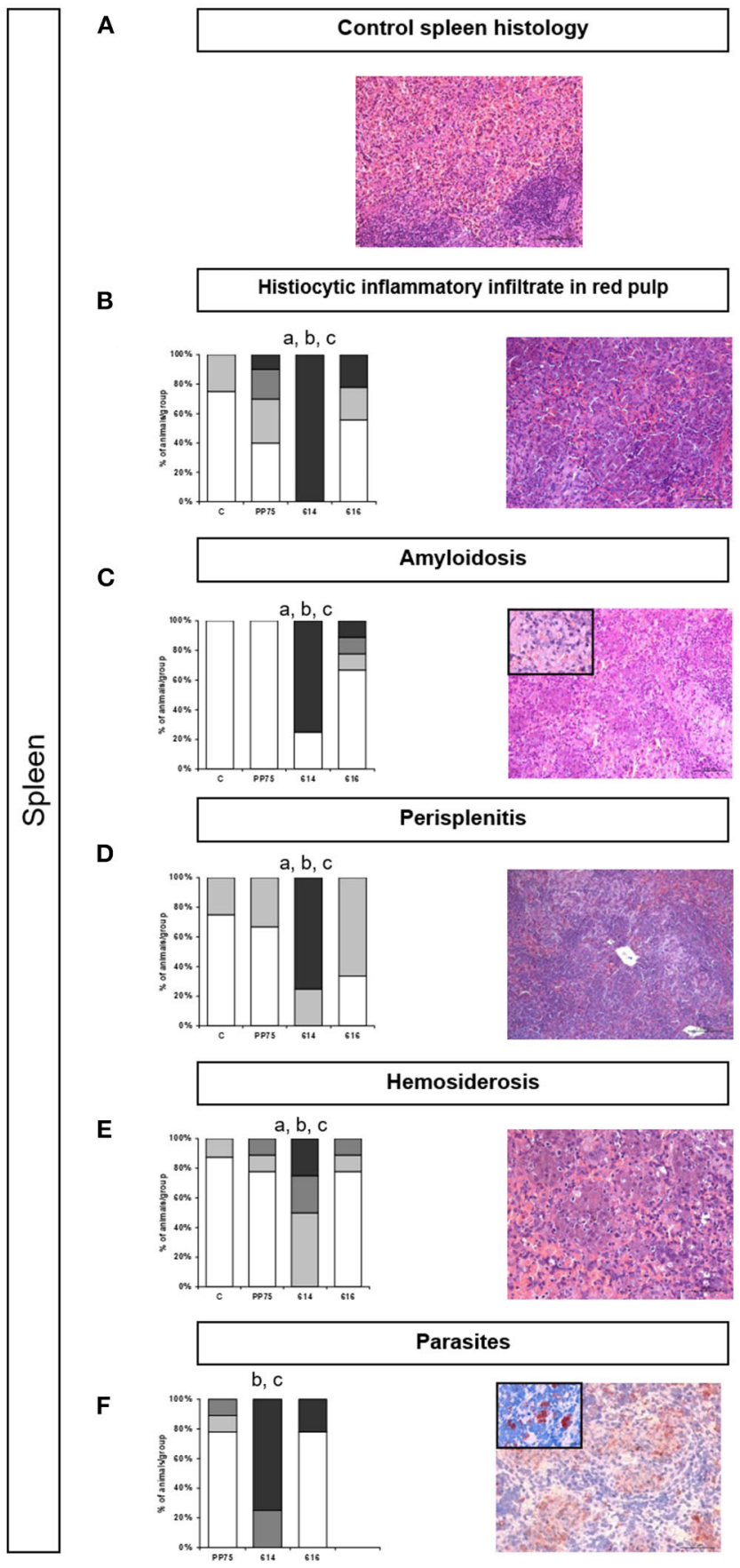

FIGURE 5 | Frequency and histopathological changes in the spleen of control group and experimentally $L$. infantum-infected groups (reference strain: PP75; and wild strains: 614 and 616). The intensity of histopathological changes was graded as absent $(-, \square)$, mild $(+, \square)$, moderate $(++, \square)$, and severe $(+++, \square$. The histopathology analysis was performed for histiocytic inflammatory infiltrate in the red pulp (B), amyloidosis (C), perisplenitis (D), hemosiderosis $€$, and parasitism (F). Images obtained from optical microscopy illustrate each analysis of HE-stained (A-E) and immunohistochemistry

(Continued) 
FIGURE 5 | (F) slides for all evaluated histopathological parameters. Representative images (B-F) correspond to animal samples from the group most affected by the infection (614). An image of the control spleen histology (A) was included to illustrate the normal histological condition in hamsters. Statistically significant differences are highlighted by the letters "a," "b," and "c" as compared to Control (C), PP75, and 616, respectively, at $P<0.05$.

lymphocytes counts in comparison to the PP75 group (Table 2).

\section{The 614 Strain Induced an Increase in the Serum AST and ALT Levels, a Clinical Marker of Hepatic Dysfunction Regarding Severe Visceral Leishmaniasis}

Biochemical evaluations consist of liver function tests, including the measurement of AST and ALT, and renal function tests based on urea and creatinine levels. Notably, animals infected with the 614 strain presented an increase $(P<0.05)$ in AST and ALT serum levels in relation to the $C$ and PP75 groups (Table 3). Moreover, the 616 strains induced increased ALT levels in comparison to the PP75 strain (Table 3).

\section{Infection Triggered by the 614 Strain Showed the Worst Histopathological Findings in Hepatic and Splenic Compartments}

In hamsters infected with the 614 strain, the intensity of inflammatory infiltrate (Figure 4B) in the liver, consisting of lymphocytes, hstiocytes, plasma cells, randomly distributed neutrophils, amyloidosis (Figure 4C), and parasitism (Figure 4D) identified by immunohistochemistry were significantly higher as compared to the other groups (C, PP75, and 616). Figure 4A represents the control group.

Similarly, in the spleen, the 614 strain induced significantly more intense histopathological changes as illustrated by high $(P<0.05)$ histiocytic infiltrate in the red pulp (Figure 5B), amyloidosis (Figure 5C), perisplenitis (Figure 5D), hemosiderosis (Figure 5E), and numbers of parasites through immunohistochemistry (Figure 5F). Figure 4A represents the control group.

\section{The 614 Strain Was Able to Infect All Hamsters According to an Analysis of the Liver and Spleen, Plus a High Parasite Load in the Liver by qPCR Analysis}

The group infected by the 614 strain presented $100 \%$ positivity in the spleen and liver compartments (Figure 6A). Moreover, the same group showed a high parasite load $(P<0.05)$ in the liver (Figure 6B) as compared to the PP75 and 616 strains. No significant changes were found in the spleen (Figure 6C).

\section{DISCUSSION}

Dogs are the main domestic reservoir of $L$. infantum favoring its transmission in urban areas $(6,7)$. In this study, we examined different $L$. infantum strains isolated from dogs living in a VL endemic area in Brazil, using in vitro and in vivo approaches to determine the infectivity and pathogenicity profiles. The wild strains used to infect canine macrophages (DH82) presented distinct virulence profiles. After in vitro infection, we selected the two strains with more and less infectivity profiles to perform the pathogenicity experiments in the hamster model. Importantly, we included a reference $L$. infantum strains (MHOM/BR/74/PP75) in all in vitro and in vivo experiments. Similarly, previous studies have used L. infantum PP75 strain as a reference strain to analyze experimental infection in hamsters $(21,22)$.

More recently, research groups have used in vitro macrophage infection as an advantageous alternative in infectivity studies $(17,18,28)$. Our previous experiments to standardize the in vitro conditions of macrophage and parasite co-cultures demonstrated that incubation after 3,24 , and $48 \mathrm{~h}$ resulted in consistent macrophage integrity and parasite growth (data not shown). However, it is important to select the best strain to use in both in vitro and in vivo experiments, considering the possibility of dichotomous virulence profiles in these two systems. Zhang and Matlashewski (29) demonstrated that the expression profile of the virulence-associated genes showed species-specific and stage-specific differences. The present study demonstrated high variability of infectivity and pathogenicity in L. infantum strains. Interestingly, the strain with less infectivity in in vitro experiments behaved differently in the in vivo analysis, leading to high infectivity and pathogenicity. The data suggests that distinct virulence patterns could occur intrinsically in the species-specific and stage-specific conditions, as recently reported for human monocytes infected with distinct Leishmania strains and species (19).

In the present experimental model, it was possible to observe that 616 was the most infective strain in the in vitro experiments, a pattern that was reversed in the in vivo experiments. Conversely, the 614 strain that presented low in vitro infectivity resulted in a high pathogenicity in hamsters. This study corroborates the data obtained by Cunha et al. (17), which showed differences between in vitro and in vivo infectivity in the murine model attributed to the intrinsic characteristics of each strain.

In vitro cultivation of Leishmania strains leads to wide variation due to laboratory conditions, becoming highly complex when comparing similar experiments. This may occur due to differences in the culture medium (30), number of passages performed, and time of the culture. Therefore, the promastigote forms generated will be differently enriched in the metacyclic forms (27), resulting in distinct infection patterns. Furthermore, the genetics and immune status of the host play an important role in the severity of the disease $(31,32)$. However, even with these variables, there is no doubt the different strains 


\section{Positivity for QPCR in the spleen and liver from experimentally L. infantum-infected groups}

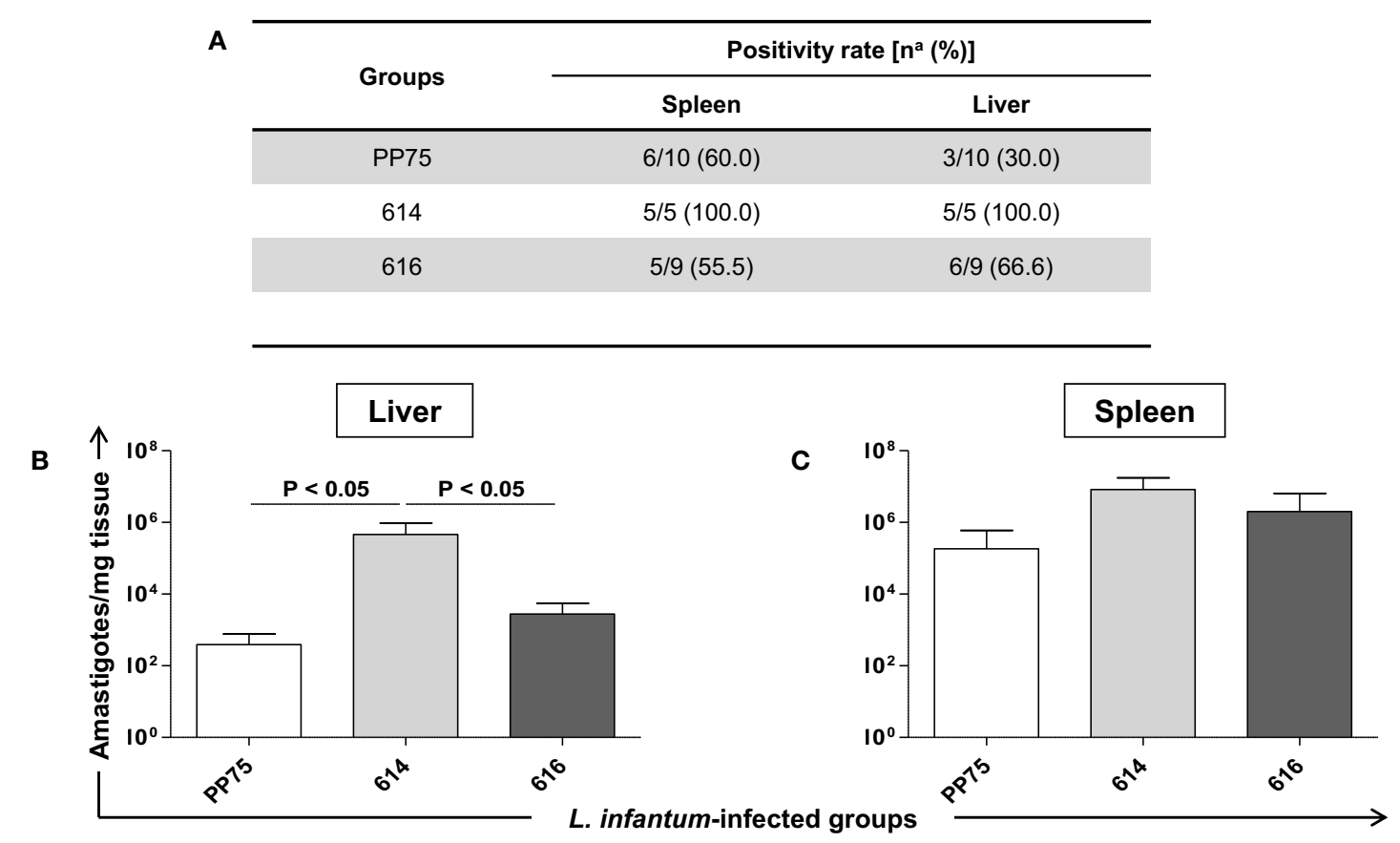

FIGURE 6 | Positivity for qPCR in the spleen and liver (A) from experimentally L. infantum-infected groups (reference strain: PP75; and wild strains: 614 and 616). (A) Number of positive hamsters in relation to total analyzed animals. Animals from $614(n=5)$ group died before the end of the experiment, as demonstrated in the survival rate displayed in Figure 2. The $y$-axis shows the number of amastigotes per milligram of tissue in the liver (B) and spleen (C). The $x$-axis shows the $L$. infantum-infected groups (reference strain: PP75 and wild strains: 614 and 616). The significant difference $(P<0.05)$ is indicated on the graph by connecting lines.

display distinct in vitro infectivity profiles, as observed in the present experiment.

The route, number of parasites, and time after infection contribute to determining the spectrum of clinical manifestation in experimental infection using the hamster model for VL $(21,22)$. The 614 strain stood out due to its high infectivity and pathogenicity in hamsters, with lymphopenia and anemia that resulted in $50 \%$ of deaths after seven months of infection. In fact, the animals of this group that died showed marked weight loss and cachexia, which lasted for a period of $\sim 1$ week until death (data not shown). Importantly, we obtained these isolates from naturally infected dogs in an endemic area and observed that the dog from which isolate 614 induced severe VL clinical signs. The dog from which isolate 616 was obtained showed few clinical signs (data not shown). These data support our hypothesis that isolate 614 is more pathogenic.

It is worth emphasizing that Leishmania-specific experimental infection in a hamster model displays many clinical pathological aspects related to canine and human VL. A number of hematological and biochemical biomarkers have been analyzed in natural and experimental VL infection as prognosis biomarkers $(21,22,25,33,34)$. In this study, hamsters infected with the
614 strain exhibited severe anemia with a reduction in red blood cells, hemoglobin, and hematocrit, along with a reduction in lymphocyte levels. Hematological parameters may have relevance in monitoring the clinical status and definition of therapeutic protocols, usually described as normocytic and normochromic anemia, displaying reduced hemoglobin and hematocrit values (35-39). Regarding the leukocytes, immunosuppression occurs with intense leukopenia, monocytopenia, lymphopenia, and eosinopenia $(11,35,38,40-44)$. Moreover, we described that hamsters infected with the 614 strain exhibited an increase in AST and ALT serum levels. Changes in the concentrations of serum aminotransferase enzymes may indicate liver cell injury (45), so these protein levels are important as a clinical VL biomarker.

In fact, the liver is one of the most affected organs in VL infection, in which the resolution of the acute infection was associated with the development of inflammatory interlobular hepatic granulomas around the Küpffer cells (46-48). According to Moreira et al. (21), increased inflammation and the presence of high numbers of granulomas may be related to intense parasitism in hamsters. Similar results were observed in hamsters infected with the 614 strain, with the liver presenting intense inflammation and amyloidosis associated with a high parasite 
load. Furthermore, it was observed that the 614 group presented intense inflammatory infiltrate in the spleen, amyloidosis, and hemosiderosis, plus a high parasite load. In contrast, the 616 strain that showed high in vitro infectivity did not present relevant clinical/pathological and biochemical/hematological findings, resulting in a mild infection with lower parasite load. Histopathology and PCR experiments agree to show mainly a higher pathogenicity caused by the isolate 614 . The differences between the parasitism evaluated by microcopy and PCR could be explained by the fact that these were different organ fragments analyzed. It is important to note that these strains were isolated from naturally L. infantum-infected dogs in an VL endemic area. Furthermore, the dogs infected with the 614 strain showed severe clinical signs indicative of VL in contrast to that observed in dogs infected with the 616 strain (data not shown). These data corroborate the high pathogenicity hypothesis related to the 614 strain. Moreover, there are studies showing that the increased gene copy numbers due to chromosome amplification may contribute to alterations in gene expression in response to environmental conditions in the host, providing a genetic basis for disease tropism (49). The analysis of L. infantum isolated from naturally infected dogs may contribute to identifying more virulent parasites, thus supporting basic and applied studies as vaccinal and therapeutic strategies. Using a genetic analysis of these strains to identify and characterize the genes expressed could contribute to identifying intrinsic parasite factors regarding distinct virulence and pathogenicity profiles.

\section{CONCLUSIONS}

This study demonstrated that L. infantum strains, obtained from naturally infected dogs, display different infectivity and pathogenicity profiles. We showed that the strain inducing the lowest infectivity profile (infection frequency and parasite load analyzed in canine macrophages) was able to induce the strongest pathogenicity (mortality, anemia, lymphopenia, clinical signs, histological damage in the liver and spleen, and parasite load in liver) in hamsters. It supports that screening of L. infantum infectivity using in vitro experiments is inadequate when selecting the best pathogenic strain.

\section{REFERENCES}

1. Carvalho EM, Teixeira RS, Johnson WD Jr. Cell-mediated immunity in American visceral leishmaniasis: reversible immunosuppression during acute infection. Infect Immunol. (1981) 33:498-500. doi: 10.1128/IAI.33.2.498-500.1981

2. Desjeux P. Leishmaniasis: current situation and new perspectives. Comp Immunol Microbiol Infect Dis. (2004) 27:305-18. doi: 10.1016/j.cimid.2004.03.004

3. Van Griensven J, Diro E. Visceral leishmaniasis. Infect Dis Clin North Am. (2012) 26:309-22. doi: 10.1016/j.idc.2012. 03.005

4. Gontijo CMF, Melo MN. Leishmaniose visceral no Brasil: quadro atual, desafios e perspectivas. Rev Brasil Epidemiol. (2004) 7:338-49. doi: 10.1590/S1415-790X2004000300011
Therefore, further studies are warranted to better understand the characteristics of these strains, allowing their use as a tool in research related to the prevention and treatment of VL.

\section{DATA AVAILABILITY STATEMENT}

All datasets generated for this study are included in the article/supplementary material.

\section{ETHICS STATEMENT}

The animal study was reviewed and approved by Ethical Committee on Animal Research of the Universidade Federal de Ouro Preto, State of Minas Gerais, Brazil (approval ID number 2012/37).

\section{AUTHOR CONTRIBUTIONS}

LR, RA-S, JC, PS, TC, and RS conceived and designed the study, contributed to the data analysis, and drafted and revised the manuscript. LR, RA-S, NM, SF, ML, JC, and FM performed the experiments. WC-V provided the 614 and 618 strains. LR, DS-L, OM-F, WC-V, WD, AR, and RG wrote and revised the manuscript. All authors contributed to the article and approved the submitted version.

\section{FUNDING}

This work was supported by Fundação de Amparo a Pesquisa do Estado de Minas (FAPEMIG) Gerais, Brazil; Conselho Nacional de Desenvolvimento Científico e Tecnológico (CNPq), Brazil, Coordenação de Aperfeiçoamento de Pessoal de Nível Superior (CAPES) and Ministério da Saúde/DECIT. RS, OM-F, WD, AR, and RG are grateful to $\mathrm{CNPq}$ for fellowships.

\section{ACKNOWLEDGMENTS}

We are grateful to Randall Hope Johnson for assistance in preparing the manuscript.
5. Harhay MO, Olliaro PL, Costa DL, Costa CH. Urban parasitology: visceral leishmaniasis in Brazil. Trends Parasitol. (2011) 27:403-9. doi: 10.1016/j.pt.2011.04.001

6. Deane LM. Reservoirs of Leishmania donovani in Brazil. Rev Assoc Med Brasil. (1961) 7:161-9.

7. Dantas-Torres F. Leishmune vaccine: the newest tool for prevention and control of canine visceral leishmaniosis and its potential as a transmission-blocking vaccine. Vet Parasitol. (2006) 141:1-8. doi: 10.1016/j.vetpar.2006.05.001

8. Palatnik-De-Sousa CB, Dos Santos WR, França-Silva JC, da Costa RT, Reis AB, Palatinik M, et al. Impact of canine control on the epidemiology of canine and human visceral leishmaniasis in Brazil. Am J Trop Med Hyg. (2001) 65:510-7. doi: 10.4269/ajtmh.2001.65.510

9. Giunchetti RC, Mayrink W, Genaro O, Carneiro CM, Corrêa-Oliveira R, Martins- Filho OA, et al. Relationship between canine visceral leishmaniosis 
and the Leishmania (Leishmania) chagasi burden in dermal inflammatory foci. J Compar Pathol. (2006) 135:100-7. doi: 10.1016/j.jcpa.2006.06.005

10. Andrade ASR, Melo MN. Canine skin and conjunctival swab samples for the detection and quantification of Leishmania infantum DNA in an endemic Urban Area in Brazil. PLoS Negl Trop Dis. (2012) 6:1596. doi: 10.1371/journal.pntd.0001596

11. Reis AB, Teixeira-Carvalho A, Giunchetti RC, Guerra LL, Carvalho MG, Mayrink W, et al. Phenotypic features of circulating leucocytes as immunological markers for clinical status and bone marrow parasite density in dogs naturally infected by Leishmania chagasi. Clin Exp Immunol. (2006) 146:303-11. doi: 10.1111/j.1365-2249.2006.03206.x

12. Ferreira RS, Ituassu LT, Almeida GG, Menezes-Souza D, Fujiwara RT, Laurenti $\mathrm{MD}$, et al. Asymptomatic dogs are highly competent to transmit Leishmania (Leishmania) infantum chagasi to the natural vector. Vet Parasitol. (2013) 23:296-300. doi: 10.1016/j.vetpar.2013.03.017

13. Sulahian A, Garin YJ, Pratlong F, Dedet JP, Derouin F. Experimental pathogenicity of viscerotropic and dermotropic isolates of Leishmania infantum from immunocompromised and immunocompetent patients in a murine model. FEMS Immunol Med Mic J. (1997) 17:131-8. doi: 10.1111/j.1574-695X.1997.tb01005.x

14. Gangneux JP, Sulahian A, Honore S, Meneceur P, Derouin F, Garin YJ. Evidence for determining parasitic factors in addition to host genetics and immune status in the outcome of murine Leishmania infantum visceral leishmaniasis. Parasite Immunol. (2000) 22:515-9. doi: 10.1046/j.1365-3024.2000.00332.x

15. Sacks DL, Modi G, Rowton E, Spath G, Epstein L, Turco SJ, et al. The role of phosphoglycans in Leishmania-sand fly interactions. Proc Natl Acad Sci USA. (2000) 97:406-11. doi: 10.1073/pnas.97.1.406

16. Wilson ME, Jeronimo SM, Pearson RD. Immunopathogenesis of infection with the visceralizing Leishmania species. Mic Pathog. (2005) 38:147-60. doi: $10.1016 /$ j.micpath.2004.11.002

17. Cunha J, Carrillo E, Sánchez C, Cruz I, Moreno J, Cordeiro-da-Silva A. Characterization of the biology and infectivity of Leishmania infantum viscerotropic and dermotropic strains isolated from HIV + and HIV- patients in the murine model of visceral leishmaniasis. Parasite Vector. (2013) 26:122. doi: 10.1186/1756-3305-6-122

18. Domínguez-Bernal G, Jiménez M, Molina R, Ordóñez-Gutiérrez L, MartínezRodrigo A, Mas A, et al. Characterisation of the ex vivo virulence of Leishmania infantum isolates from Phlebotomus perniciosus from an outbreak of human leishmaniosis in Madrid, Spain. Parasit Vectors. (2014) 7:499. doi: 10.1186/s13071-014-0499-1

19. Viana AG, Magalhães LMD, Giunchetti RC, Dutra WO, Gollob KJ. Infection of human monocytes with Leishmania infantum strains induces a down modulated response when compared with infection with Leishmania braziliensis. Front Immunol. (2018) 8:1896. doi: 10.3389/fimmu.2017. 01896

20. Coura-Vital W, Marques MJ, Veloso VM, Roatt BM, AguiarSoares RD, Reis LE, et al. Prevalence and factors associated with Leishmania infantum infection of dogs from an urban area of Brazil as identified by molecular methods. PLoS Negl Trop Dis. (2011) 5:e1291. doi: 10.1371/journal.pntd.0001291

21. Moreira N, Vitoriano-Souza J, Roatt BM, Vieira PMA, Ker HG, de Oliveira Cardoso JM, et al. Parasite burden in hamsters infected with two different strains of Leishmania (Leishmania) infantum: "Leishman Donovan units" versus real-time PCR. PLoS ONE. (2012) 7:e47907. doi: 10.1371/journal.pone.0047907

22. Moreira ND, Vitoriano-Souza J, Roatt BM, Vieira PM, Coura-Vital W, Cardoso JM, et al. Clinical, hematological and biochemical alterations in hamster (Mesocricetus auratus) experimentally infected with Leishmania infantum through different routes of inoculation. Parasite Vector. (2016) 9:181. doi: 10.1186/s13071-016-1464-y

23. Viana KF, Aguiar-Soares RD, Ker HG, Resende LA, Souza-Fagundes EM, Dutra WO, et al. Setting the proportion of CD4+ and CD8+ Tcells co-cultured with canine macrophages infected with Leishmania chagasi. Vet Parasitol. (2015) 211:124-32. doi: 10.1016/j.vetpar.2015. 05.025
24. Gonçalves R, Vieira ER, Melo MN, Gollob KJ, Mosser DM, Tafuri WL. A sensitive flow cytometric methodology for studying the binding of $L$. chagasi to canine peritoneal macrophages. BMC Infect Dis. (2005) 24:5-39. doi: 10.1186/1471-2334-5-39

25. Gifawesen C, Farrell JP. Comparison of T-cell responses in self-limiting versus progressive visceral Leishmania donovani infections in golden hamsters. Infect Immun. (1989) 56:3091-6. doi: 10.1128/IAI.57.10.3091-3096.1989

26. Tafuri WL, Santos RL, Arantes RM, Gonçalves R, de Melo MN, Michalick MS, et al. An alternative immunohistochemical method for detecting Leishmania amastigotes in paraffin-embedded canine tissues. J Immunol Met. (2004) 292:17-23. doi: 10.1016/j.jim.2004.05.009

27. Bretagne S, Durand R, Olivi M, Sulahian A, Rivollet D, Vidaud M, et al. Real-time PCR as a new tool for quantifying Leishmania infantum in liver in infected mice. Clin Diagn Lab Immunol. (2001) 8:828-31. doi: 10.1128/CDLI.8.4.828-831.2001

28. Maia C, Nunes M, Cristóvão J, Campino L. Experimental canine leishmaniasis: clinical, parasitological and serological follow-up. Acta Trop. (2007) 116:193-9. doi: 10.1016/j.actatropica.2010.08.001

29. Zhang WW, Matlashewski G. Screening Leishmania donovani-specific genes required for visceral infection. Mol Microbiol. (2010) 77:505-17. doi: $10.1111 / j .1365-2958.2010 .07230 . x$

30. Dey T, Afrin F, Anam K, Ali N. Infectivity and virulence of Leishmania donovani promastigotes: a role for media, source, and strain of parasite. $J$ Eukaryot Microbiol. (2002) 49:270-4. doi: 10.1111/j.1550-7408.2002.tb00369.x

31. Gradoni L, Gramiccia M. Leishmania infantum tropism: strain genotype or host immune status? Parasitol Today. (1994) 10:264-7. doi: 10.1016/0169-4758(94)90142-2

32. Rivas L, Moreno J, Cañavate C, Alvar J. Virulence and disease in Leishmaniasis: what is relevant for the patient? Trends Parasitol. (2004) 20:297-301. doi: 10.1016/j.pt.2004.05.005

33. Bunn-Moreno MM, Madeira ED, Miller K, Menezes JA, Campos-Neto A. Hypergammaglobulinaemia in Leishmania donovani infected hamsters: possible association with a polyclonal activator of $B$ cells and with suppression of T cell function. Clin Exp Immunol. (1985) 59:427-34.

34. Mathias R, Costa FA, Goto H. Detection of immunoglobulin G in the lung and liver of hamsters with visceral leishmaniasis. Braz J Med Biol Res. (2001) 34:539-43. doi: 10.1590/S0100-879X2001000400015

35. Costa-Val AP, Cavalcanti RR, de Figueiredo Gontijo N, Michalick MS, Alexander B, Willians P, et al. Canine visceral leishmaniasis: relationships between clinical status, humoral immune response, haematology and Lutzomyia (Lutzomyia) longipalpis infectivity. Vet J. (2007) 174:636-43. doi: $10.1016 /$ j.tvjl.2006.11.006

36. Reis AB, Martins-Filho OA, Teixeira-Carvalho A, Giunchetti R, Carneiro CMC, Mayrink W, et al. Systemic and compartmentalized immune responses in canine visceral Leishmaniasis. Vet Immunol Immunopathol. (2009) 128:8795. doi: 10.1016/j.vetimm.2008.10.307

37. Solano-Gallego L, Koutinas A, Miró G, Cardoso L, Pennisi MG, Ferrer L, et al. Directions for the diagnosis, clinical staging, treatment and prevention of canine leishmaniasis. Vet Parasitol. (2009) 165:1-18. doi: 10.1016/j.vetpar.2009.05.022

38. Oliveira JM, Fernandes AC, Dorval ME, Alves TP, Fernandes TD, Oshiro ET, et al. Mortality due to visceral leishmaniasis: clinical and laboratory characteristics. Rev Soc Brasil Med Trop. (2010) 43:188-93. doi: 10.1590/S0037-86822010000200016

39. Brasil. Guia de Vigilância Epidemiológica, Secretaria de Vigilância em Saúde. Brasília: Departamento de Vigilância Epidemiológica, Ministério da Saúde (2009).

40. Pearson $\mathrm{RD}$, Sousa AQ. Clinical spectrum of Leishmaniasis. Clin Infect Dis. (1996) 22:1-13. doi: 10.1093/clinids/22.1.1

41. Bourdoiseau G, Bonnefont C, Magnol JP, Saint-André I, Chabanne L. Lymphocyte subset abnormalities in canine leishmaniasis. Vet Immunol Immunopathol. (1997) 56:345-51. doi: 10.1016/S0165-2427(96)05768-6

42. Ciaramella P, Oliva G, Luna RD, Gradoni L, Ambrosio R, Cortese L, et al. A retrospective clinical study of canine leishmaniasis in 150 dogs naturally infected by Leishmania infantum. Vet Rec. (1997) 141:539-43. doi: $10.1136 / v r .141 .21 .539$ 
43. Pastorino AC, Jacob CM, Oselka GW, Carneiro-Sampaio MM. Visceral leishmaniasis: clinical and laboratorial aspects. J Ped. (2002) 78:120-7. doi: $10.2223 /$ JPED.820

44. Pedrosa CMS. Aspectos clínicos e epidemiológicos da leishmaniose visceral em menores de 15 anos procedentes de Alagoas. Brasil Rev Soc Brasil Med Trop. (2004) 37:300-4. doi: 10.1590/S0037-868220040004 00003

45. Kaneko JJ, Harvey JW, Bruss ML. Clinical Biochemestry of Domestic Animals. 1st ed. San Diego: Academic Press Inc. (1997).

46. Wilson ME, Innes DJ, Sousa AD, Pearson RD. Early histopathology of experimental infection with Leishmania donovani in hamsters. J Parasitol. (1987) 73:55-63. doi: 10.2307/3282344

47. Vianna VL, Takiya CM, de Brito-Gitirana L. Histopathologic analysis of hamster hepatocytes submitted to experimental infection with Leishmania donovani. Parasitol Res. (2002) 88:829-36. doi: 10.1007/s00436-001-0577-0

48. Stanley AC, Engewerda CR. Balancing immunity and pathology in visceral leishmaniasis. Immunol Cell Biol. (2007) 85:138-47. doi: $10.1038 /$ sj.icb7100011
49. Rogers MB, Hilley JD, Dickens NJ, Wilkes J, Depledge DP, Harris D, et al Chromosome and gene copy number variation allow major structural change between species and strains of Leishmania. Genome Res. (2011) 21:2129-42. doi: $10.1101 /$ gr.122945.111

Conflict of Interest: The authors declare that the research was conducted in the absence of any commercial or financial relationships that could be construed as a potential conflict of interest.

Copyright (c) 2020 Resende, Aguiar-Soares, Moreira, Ferreira, Lanna, Cardoso, Mathias, Coura-Vital, Mariano, Leite, Silveira, de Carvalho, Santos, SilveiraLemos, Martins-Filho, Dutra, Reis and Giunchetti. This is an open-access article distributed under the terms of the Creative Commons Attribution License (CC BY). The use, distribution or reproduction in other forums is permitted, provided the original author(s) and the copyright owner(s) are credited and that the original publication in this journal is cited, in accordance with accepted academic practice. No use, distribution or reproduction is permitted which does not comply with these terms. 